\title{
Nuclear expression of $Y$ box binding-1 is important for resistance to chemotherapy including gemcitabine in TP53-mutated bladder cancer
}

\author{
TAKAHISA YAMASHITA ${ }^{1}$, MORIHIRO HIGASHI $^{1}$, SHUJI MOMOSE $^{1}$, \\ MAKOTO MOROZUMI ${ }^{2}$ and JUN-ICHI TAMARU ${ }^{1}$ \\ Departments of ${ }^{1}$ Pathology and ${ }^{2}$ Urology, Saitama Medical Center, Saitama Medical University, \\ 1981 Kamoda, Kawagoe-shi, Saitama 350-8550, Japan
}

Received February 2, 2017; Accepted May 26, 2017

DOI: 10.3892/ijo.2017.4031

\begin{abstract}
The development and acquisition of multiple drug resistance in cancer cells remain a major obstacle in the treatment of bladder cancer. Nuclear translocation of Y box binding-1 (YB-1), which is a member of a family of DNA-binding proteins that contain a cold shock domain, plays a significant role in the acquisition of drug resistance by upregulating expression of the multidrug resistance-1 $(M D R-1)$ gene product, P-glycoprotein. The tumor suppressor protein p53 is thought to be essential for nuclear translocation of YB-1. We hypothesized that nuclear translocation of YB-1 might be associated with drug resistance of bladder cancer with an abnormality of the TP53 gene that results in a mutated p53 protein. To test this hypothesis, we analyzed the association of YB-1 with drug resistance of TP53-mutated bladder cancer, including immunohistochemical analysis of YB-1, P-glycoprotein and p53 in vivo as well as the function of YB-1 nuclear translocation and regulation of its translocation by p53 in vitro. Additionally, we examined the association between the nuclear translocation of YB-1 and gemcitabine, a major anticancer-drug for bladder cancer, in cancer cell lines. Nuclear expression of YB-1 was correlated with the expression of $\mathrm{P}$-glycoprotein and p53 in bladder cancer cases $(\mathrm{P}<0.05)$. In vitro, both introduction of TP53 and gemcitabine induced
\end{abstract}

Correspondence to: Dr Jun-Ichi Tamaru, Department of Pathology, Saitama Medical Center, Saitama Medical University, 1981 Kamoda, Kawagoe-shi, Saitama 350-8550, Japan

E-mail: jtamaru@saitama-med.ac.jp

Abbreviations: BCG, Bacillus Calmette-Guérin; CE, cytosol extraction; CIS, carcinoma in situ; GC, gemcitabine+cisplatin; mCherry-YB-1, mCherry-fused YB-1; MDR-1, multidrug resistance-1; N/C ratio, nuclear/cytosolic ratio; ROI, region of interest; YB-1, Y box binding-1

Key words: bladder cancer, CIS, YB-1, P-glycoprotein, p53, gemcitabine nuclear translocation of YB-1. These data indicate that YB-1 translocates to the nucleus coordinately with p53 expression and is involved in gemcitabine resistance in bladder cancer. Nuclear expression of YB-1 is important for resistance to chemotherapy including gemcitabine in TP53-mutated bladder cancer.

\section{Introduction}

Bladder cancer is thought to develop along two major, independent pathways and these progression pathways have different genetic abnormalities (1). One is the 'papillary pathway' that is characterized as low-grade papillary cancer via epithelial hyperplasia. Approximately $80 \%$ of bladder cancers arise from the papillary pathway and these cancers can be treated by transurethral resections and have a relatively good prognosis compared to other types. This pathway is associated with genetic abnormalities such as activating mutations of the oncogene, HRAS and the receptor tyrosine kinase gene, FGFR3. The other pathway is the 'carcinoma in situ (CIS) pathway'. This pathway is associated with genetic abnormalities of the tumor suppressor genes, TP53 and $R b$. Approximately $80 \%$ of muscle-invasive bladder cancer is thought to arise from this pathway. CIS is a distinct entity with a high-grade malignancy and a high tendency to progress to muscle-invasive bladder cancer (2). CIS is commonly treated by intravesical infusion therapy. Although therapy has been developed for CIS, there remain therapy-resistant cases in $\sim 30 \%$ of patients treated with Bacillus Calmette-Guérin (BCG) therapy and in $50 \%$ of cases treated with intravesical chemotherapy (3). Moreover, in more than $50 \%$ of cases, residual CIS lesions are found in radical cystectomy specimens after preoperative chemotherapy (4). Thus, CIS is considered to have the potential to develop anticancer drug resistance.

Recently, gemcitabine combined with cisplatin(GC) therapy has been accepted as a new standard treatment for advanced bladder cancer because of fewer adverse events than those with methotrexate, vinblastine, doxorubicin and cisplatin (MVAC) therapy (5). However, despite a reasonable response rate after initial chemotherapy, $60-70 \%$ of patients relapse within the first year probably because of drug resistance to gemcitabine. 
The mechanism of drug resistance to gemcitabine has not been elucidated. Recently, gemcitabine had been used not only as a systemic chemotherapy for metastatic bladder cancer, but also as intravesical therapy for CIS (6). It is therefore necessary to clarify the mechanism of CIS drug-resistance to gemcitabine, which could provide a basis for the development of novel strategies for bladder cancer.

Y box binding-1 (YB-1) is a member of a family of DNA-binding proteins that contain a cold shock domain and it is directly involved in the cellular response to genotoxic stress, such as DNA-damaging agents and UV irradiation (7-9). YB-1 is predominantly localized to the cytoplasm in many cancer cells but its translocation to the nucleus is directly induced by phosphorylated $\mathrm{Akt}$, in response to these environmental stresses (10-14). Nuclear translocated YB-1 upregulates transcription of the multidrug resistance-1 (MDR-1) gene. YB-1 associates with p53 and p53 is thought to be essential for nuclear translocation of YB-1 (15-17).

In the present study, we aimed to analyze YB-1 association with the drug resistance of TP53-mutated bladder cancer, including immunohistochemical analysis of YB-1, $\mathrm{P}$-glycoprotein and $\mathrm{p} 53$ in vivo and analysis of the role of $\mathrm{p} 53$ in the nuclear translocation and nuclear function of YB-1 in vitro. Additionally, we examined the association between gemcitabine and the nuclear translocation of YB-1.

\section{Materials and methods}

Patients. Transurethral resected specimens, and clinical data were obtained retrospectively from 81 patients newly diagnosed with CIS from 2000 to 2013 at the Department of Pathology of Saitama Medical Center, Saitama Medical University. The examined specimens were from patients previously untreated for CIS. The staging was performed according to the TNM classification (7th edition). All specimen collections were approved by the ethics committee of Saitama Medical Center, Saitama Medical University (no. 992).

Immunohistochemical analysis of clinical samples. The following antibodies were used for immunohistochemical detection; anti-YB-1 mouse monoclonal IgG antibody (clone: 21A3, 1:100; Immuno-Biological Laboratories, Gunma, Japan), anti-p53 mouse monoclonal $\mathrm{IgG}$ antibody (clone: DO-7, 1:40; Dako, Tokyo, Japan) and anti-P-glycoprotein mouse monoclonal IgG antibody (clone: C494, 1:100; Thermo Fisher Scientific, Waltham, MA, USA) antibodies. Immunohistochemical staining for p53 was performed using the Ventana iVIEW DAB kit reagents (Ventana Medical Systems, Inc., Tucson, AZ, USA) and an auto-immunostainer (Ventana ULTRA). For YB-1 and P-glycoprotein detection, the BOND polymer system refine kit and an auto-immunostainer (BOND III; Leica Microsystems GmbH, Wetzlar, Germany) were used according to the manufacturer's instructions. Protein expression was blindly assessed by two pathologists (T.Y. and J.T.). Expression of YB-1 and p53 was categorized into two groups according to the proportion of expressing cells in 5 hot spots of the specimens: positive, $\geq 30 \%$ of cancer cells stained; negative, $<30 \%$ of cancer cells stained. Nuclear expression of YB-1 was evaluated according to the presence or absence of staining in the nucleus. For
P-glycoprotein expression, positive staining of $\geq 50 \%$ of the cells was defined as positive.

Cell culture, reagents and materials. The human cervical cancer cell line HeLa, the human breast cancer cell line MCF-7 and the human skin cancer cell line A431 were obtained from the American Type Culture Collection (ATCC; Rockville, MD, USA). The human bladder cancer cell lines, 5637 and T24 were obtained from the RIKEN BioResource Center (Saitama, Japan). HeLa, MCF-7, T24 and A431 cells were maintained in Dulbecco's modified Eagle's medium (DMEM; Thermo Fisher Scientific) and 5637 cells were maintained in RPMI-1640 medium (Thermo Fisher Scientific). Media were supplemented with $10 \%$ fetal bovine serum (FBS; Nichirei Biosciences, Inc., Tokyo, Japan), L-glutamine and antibiotics (penicillin and streptomycin). Gemcitabine was purchased from Taiho Pharmaceutical, Co., Ltd. (Tokyo, Japan) and cisplatin was purchased from Nichi-Iko Pharmaceutical, Co., Ltd. (Toyama, Japan).

Plasmids. Total RNA was extracted from cells using TRIzol reagent (Thermo Fisher Scientific) and reverse transcribed using the SuperScript ${ }^{\mathrm{TM}}$ III First-Strand Synthesis SuperMix (Thermo Fisher Scientific) for polymerase chain reaction (PCR), according to the manufacturer's protocol. YB-1 and p53 cDNA sequence was obtained from the National Center for Biotechnology Information (NCBI) GeneBank database (http: //www. ncbi.nlm.nih.gov/genbank/). The cDNAs encoding YB-1 and wild-type p53 were cloned using PCR of normal lymphocyte mRNA from a healthy donor, and that encoding mutant p53 (R273H) was cloned by PCR of the mRNA from A431 cells. The primers for PCR were: YB-1 sense, 5'-GGACTCAGATCTCGAGCAACCATGAGCAGC GAGG-3' and antisense, 5'-GTCGACTGCAGAATTTTTAC TCAGCCCCGCCCTGC-3'; p53 sense, 5'-GGACTCAGAT CTCGAGCCATGGAGGAGCCGC-3' and antisense, 5'-GTC GACTGCAGAATTTGTCAGTCTGAGTCAGGCCCT-3'. PCR was perfomed with an initial denaturation step at $98^{\circ} \mathrm{C}$ for $1 \mathrm{~min}$, followed by 30 cycles of denaturation at $98^{\circ} \mathrm{C}$ for $10 \mathrm{sec}$, annealing at $55^{\circ} \mathrm{C}$ for $15 \mathrm{sec}$ and extension at $68^{\circ} \mathrm{C}$ for 90 sec. The PCR fragments from p53 and YB-1 cDNAs were cloned in frame with the enhanced green fluorescence protein (EGFP) or mCherry (Clontech Laboratories, Inc., Mountain View, CA, USA) as a C-terminal fusion using In-Fusion HD cloning kit (Clontech Laboratories). The insert sites were released from EGFP and mCherry by digestion with XhoI and EcoRI. Transfections were performed with Lipofectamine 3000 (Thermo Fisher Scientific) as directed by the manufacturer.

Small interfering RNAs (siRNA). Two siRNA species for knockdown of YB-1, YB-1 siRNA I (cat. no. \#6206) and II (cat. no. \#6207), as well as control siRNA (cat. no. \#6568), were purchased from Cell Signaling Technology (Danvers, MA, USA). Transfections were performed with Lipofectamine 2000 (Thermo Fisher Scientific) as directed by the manufacturer.

Gemcitabine cytotoxicity assay. The 5637 cells transfected with YB-1 siRNA or control siRNA were incubated for 
$72 \mathrm{~h}$ with $100 \mathrm{nM}$ of gemicitabine. Viable cells were labeled using the Cell Counting kit-8 (CCK-8; Dojindo Molecular Technologies Inc., Kumamoto, Japan) and were quantified using a microplate spectrophotometer (Bio-Rad Laboratories, Inc., Tokyo, Japan). Cell viability was determined according to the manufacturer. One untreated control sample was used for each sample.

Cell imaging. At 20 to $48 \mathrm{~h}$ after transfection the cells were imaged using an inverted fluorescent microscope (TE2000-S Eclipse; Nikon, Corp., Tokyo, Japan) equipped with a cooled CCD camera (CoolSNAP HQTM ; Roper Scientific $\mathrm{GmbH}$, Ottobrunn, Germany), with a precentered fiber illuminator as a light source (Intensilight C-HGFI; Nikon) and controlled by Image-Pro Plus ${ }^{\circledR}$ software (Media Cybernetics, Inc., Rockville, MD, USA). Oil immersion objective lenses of x40 or x100 were used for all imaging. For immunofluorescence analysis of YB-1 in cells, the cells were incubated with antiYB-1 antibody for $1 \mathrm{~h}$ after fixation in $4 \%$ paraformaldehyde for $10 \mathrm{~min}$ and permeabilization in $0.2 \%$ Triton X-100 for 10 min. Conjugated antibody was visualized with Flour Alexa 594-conjugated anti-mouse secondary antibody (Cell Signaling Technology).

Image analysis. Obtained images were analyzed with Fiji software (https://fiji.sc/). The nuclear/cytosolic (N/C) ratio of fluorescence intensity in the region of interest (ROI) of cells was calculated from $\sim 100$ cells.

Nuclear/cytosolic protein extraction and western blotting. For preparation of nuclear and cytosolic extracts, cells were suspended in $350 \mu \mathrm{l}$ of cytosol extraction (CE) buffer $(10 \mathrm{mM}$ HEPES pH 7.6, $60 \mathrm{mM} \mathrm{KCl,} 1 \mathrm{mM}$ EDTA, 0.075\% NP-40, $1 \mathrm{mM}$ dithiothreitol, protease inhibitor cocktail), incubated on ice for $3 \mathrm{~min}$ and centrifuged at 1,500 $\mathrm{x} \mathrm{g}$ for $4 \mathrm{~min}$ (18). After removal of the supernatant (cytosolic extract), the pellet was obtained as a nuclear extract after two washes in $1 \mathrm{ml}$ of CE buffer. Nuclear and cytosolic extracts were dissolved in $120 \mu \mathrm{l}$ of 1x Laemmli buffer. For whole protein extracts, cells were suspended in 1x Laemmli buffer, followed by SDS-PAGE and western blotting. Protein concentrations were determined using Pierce BCA Protein assay kit (Thermo Fisher Scientific) according to the standard protocol of the manufacturer. Equal amounts of protein (20-40 $\mu \mathrm{g}$ protein/lane) were separated on a $6-12 \%$ sodium dodecyl sulfate (SDS) gel via polyacrylamide gel electrophoresis (PAGE) and transferred onto polyvinylidene difluoride (PVDF) membranes. The primary antibodies used for western blotting were as follows; anti-YB-1 mouse monoclonal IgG antibody (clone: 21A3, 1:100), anti-phosphoYB-1 rabbit monoclonal IgG antibody (clone: 34A2, 1:100; Cell Signaling Technology), anti-P-glycoprotein mouse monoclonal IgG antibody (sc-55510; Santa Cruz Biotechnology, Dallas, TX, USA), anti-p53 mouse monoclonal IgG antibody (clone: DO-7, 1:200; Dako), anti-Akt goat polyclonal IgG antibody (clone: C-20, 1:2,000; Santa Cruz Biotechnology), anti-phospho-Akt rabbit monoclonal IgG antibody (clone: D9E, 1:1,000; Cell Signaling Technology), anti-Fibrillarin rabbit monoclonal $\mathrm{IgG}$ antibody (clone: 13C3, 1:1,000; Cell Signaling Technology), anti- $\alpha / \beta$ tubulin rabbit polyclonal IgG antibody (1:1,000; Cell Signaling Technology), and anti-actin
Table I. Clinicopathological features and immunohistochemical data of the study patients.

\begin{tabular}{lc}
\hline Characteristics & No. of patients $(\%)$ \\
\hline All patients & $81(100)$ \\
Age (years) & \\
$<70$ & $47(58)$ \\
$\geq 70$ & $34(42)$ \\
Sex & \\
Male & $71(88)$ \\
Female & $10(12)$ \\
Stage & \\
pTis & $78(96)$ \\
pTis+pTa & $3(4)$ \\
Immunohistochemistry & \\
YB-1 & \\
Positive & $52(64)$ \\
Nuclear positive & $19(23)$ \\
Cytoplasmic positive & $37(46)$ \\
Negative & $29(36)$ \\
p53 & \\
Positive & $41(51)$ \\
Negative & $40(49)$ \\
P-glycoprotein & \\
Positive & $31(38)$ \\
Negative & \\
\hline & \\
&
\end{tabular}

goat polyclonal IgG antibody (clone: I-19, 1:1,000; Santa Cruz Biotechnology). Conjugated antibodies were detected by the appropriate secondary antibody: mouse IgG HRP-linked (1:5,000; Cell Signaling Technology) or rabbit IgG HRP-linked (1:5,000; Cell Signaling Technology) antibody or Peroxidase AffiniPure $\mathrm{F}\left(\mathrm{ab}^{\prime}\right)_{2}$ Fragment rabbit anti-goat IgG (1:5,000; JIR, West Grove, PA, USA). Bands were visualized using the Lumi cube (Liponics, Ltd., Tokyo, Japan).

Statistical analysis. Comparison between the two groups of the immunohistochemical analysis was made using Fisher's exact test. The N/C ratio and viability of cells were analyzed using a Wilcoxon signed-rank test or a Steel's test. In all cases, results were considered significant at $\mathrm{P}<0.05$. Statistical testing was performed using JMP 10 (SAS Institute Japan Ltd., Tokyo, Japan).

\section{Results}

Localization of $Y B-1$ and expression of 533 and $P$-glycoprotein in CIS. We first investigated the expression of YB-1 and P-glycoprotein in CIS (pTis) lesions in newly diagnosed bladder cancer samples. Table I summarizes the characteristics of the patients. Seventy-one $(88 \%)$ out of the 81 patients were male and ten patients $(12 \%)$ were female. Lesions of non-invasive papillary carcinoma ( $\mathrm{pTa}$ ) were also observed in 3 patients (4\%) whereas 78 patients $(96 \%)$ had a pTis lesion only. YB-1 
A

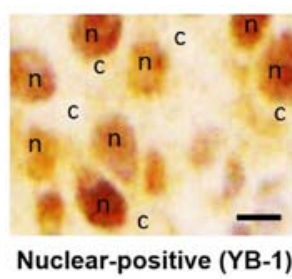

C

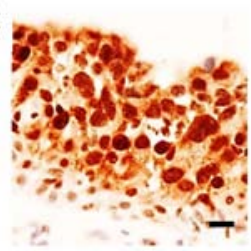

YB-1

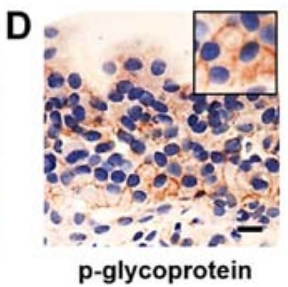

p-glycoprotein

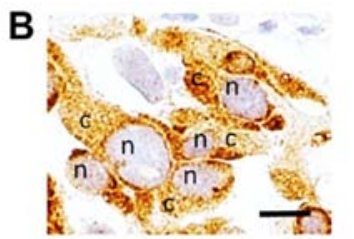

Cytoplasmic-positive (YB-1)

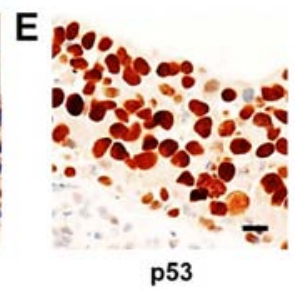

Figure 1. Immunohistochemical analysis of Y box binding-1 (YB-1), p53 and P-glycoprotein expression in carcinoma in situ (CIS) cases. Representative immunohistochemical features of YB-1 in CIS tissues. (A) Nuclear expression, (B) cytoplasmic expression of YB-1. Immunohistochemical analysis of (C) YB-1, (D) P-glycoprotein and (E) p53 in the same specimen of a CIS case. Nuclear expression of YB-1 and p53, cell membranous expression of P-glycoprotein (inset with a 2-fold magnification) was observed in the tissue of a patient without anticancer therapy. Micrographs were taken at x400 magnification. Bars, $10 \mu \mathrm{m}$. n, nucleus; c, cytoplasm.

Table II. Correlations between expression of nuclear YB-1, P-glycoprotein and p53 in carcinoma in situ cases.

\begin{tabular}{|c|c|c|c|}
\hline & \multicolumn{2}{|c|}{ P-glycoprotein } & \multirow[b]{2}{*}{ P-value } \\
\hline & Negative (\%) & Positive (\%) & \\
\hline \multicolumn{4}{|l|}{ Nuclear YB-1 } \\
\hline Negative $(n=62)$ & $42(68)$ & $20(32)$ & $<0.05$ \\
\hline \multirow[t]{3}{*}{ Positive $(n=19)$} & $7(37)$ & $12(63)$ & \\
\hline & \multicolumn{2}{|c|}{ Nuclear YB-1 } & \\
\hline & Negative (\%) & Positive (\%) & P-value \\
\hline \multicolumn{4}{|l|}{ p53 } \\
\hline Negative $(n=40)$ & $38(95)$ & $2(5)$ & $<0.05$ \\
\hline \multirow[t]{3}{*}{ Positive $(n=41)$} & $24(59)$ & $17(41)$ & \\
\hline & \multicolumn{2}{|c|}{ P-glycoprotein } & \\
\hline & Negative (\%) & Positive (\%) & P-value \\
\hline \multicolumn{4}{|l|}{ p53 } \\
\hline Negative $(n=40)$ & $29(73)$ & $11(27)$ & $<0.05$ \\
\hline Positive $(n=41)$ & $20(49)$ & $21(51)$ & \\
\hline
\end{tabular}

was expressed in 52 cases (64\%). Nuclear expression of YB-1 was observed in 19 cases $(23 \%)$, cytoplasmic expression in 37 cases $(46 \%)$ and both nuclear and cytoplasmic expression was observed in 4 cases (5\%) (Fig. 1A and B). P-glycoprotein expression was observed in 31 cases $(38 \%)$. Expression of p-glycoprotein was significantly correlated with nuclear expression of YB-1 ( $\mathrm{P}<0.05$; Table I and Fig. $1 \mathrm{C}$ and D). Next, to clarify the involvement of p53, we investigated

A

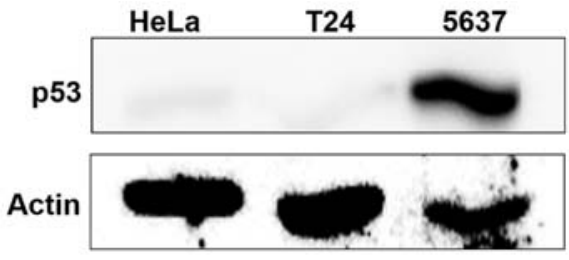

B

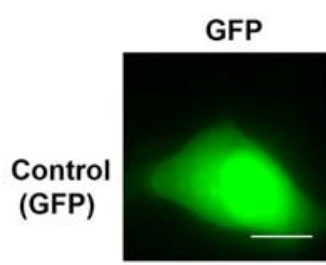

HeLa

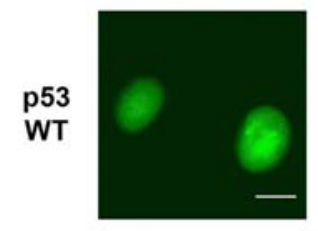

YB-1

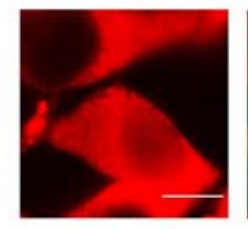

Merged
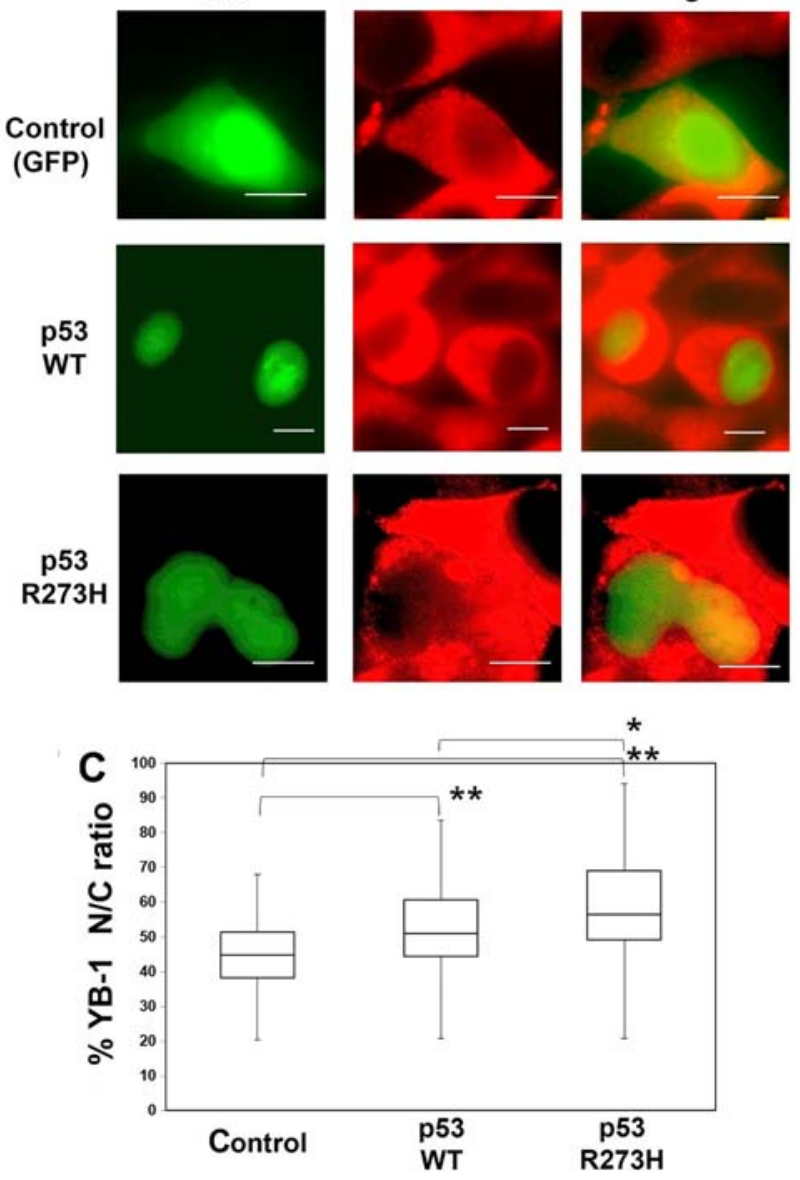

Figure 2. p53 expression in the cancer cell lines and introduction of p53 causes nuclear translocation of endogenous YB-1 in vitro. (A) p53 expression in the cancer cell lines was analyzed by western blotting. Western blotting showed little or no p53 expression in HeLa or T24 cells and high p53 expression in 5637 cells. (B) HeLa cells were transfected with pEGFP alone (Control), pEGFP-wild type-p53 (p53-WT) or pEGFP-mutant-p53 (p53-R273H). Approximately $36 \mathrm{~h}$ later, the cells were fixed and stained with an anti-YB-1 antibody (red). Representative immunofluorescent images are shown. The merged image (right) shows colocalization of p53 (left column, middle and bottom rows) and YB-1 (middle column). YB-1 nuclear translocation was observed more clearly in cells transfected with p53-R273H (bottom row, nuclear YB-1 is yellow in the merged image) than in the other cells. (C) Nuclear/cytoplasmic (N/C) ratio of YB-1 in each of the transfected cell lines. The N/C ratio of HeLa cells transfected with p53-WT or R273H was significantly increased compared with control. The middle line of the box is the median. Brackets with asterisks indicate statistically significant differences between the data sets based on a Wilcoxon signed-rank test. ${ }^{*} \mathrm{P}<0.05,{ }^{* *} \mathrm{P}<0.01$

the expression of p53 in pTis cancers. p53 was expressed in 41 cases $(51 \%)$ of pTis lesions (Fig. 1E) and its expression correlated with the nuclear translocation of YB-1 $(\mathrm{P}<0.05$; Table II) and with the expression of $\mathrm{P}$-glycoprotein $(\mathrm{P}<0.05$; Table II). These data suggested that YB-1 coordinately 
A a

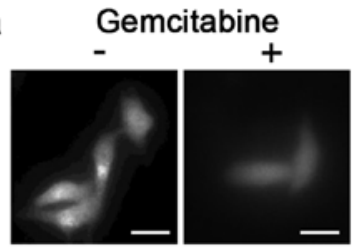

HeLa (mCherry alone) b

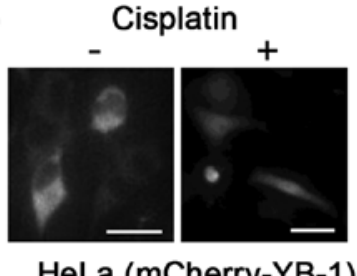

HeLa (mCherry-YB-1)

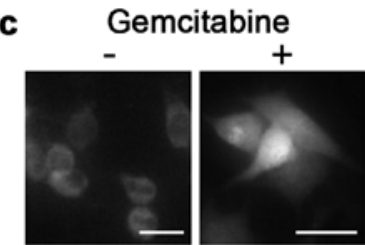

HeLa (mCherry-YB-1)

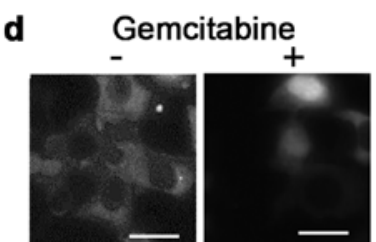

MCF-7 (mCherry-YB-1)

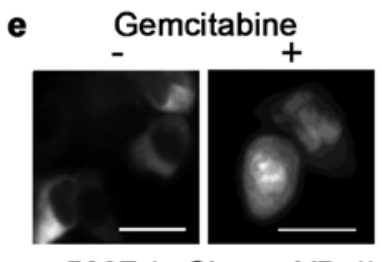

5637 (mCherry-YB-1)

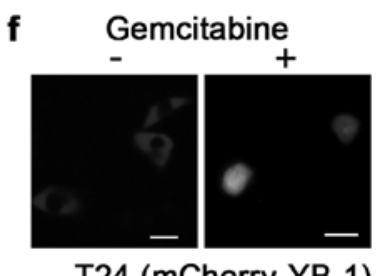

T24 (mCherry-YB-1)

B

5637

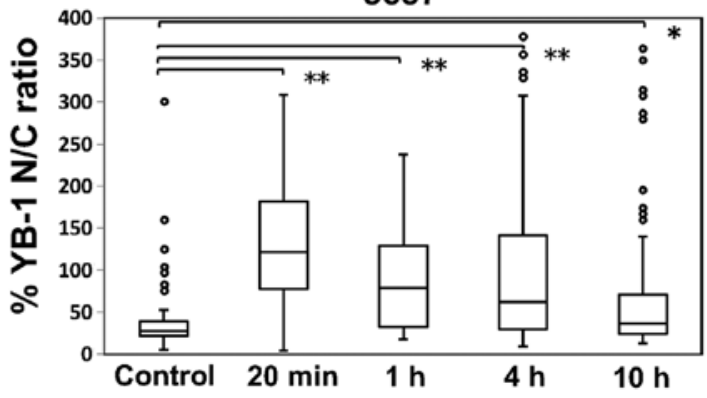

Figure 3. Gemcitabine treatment induces nuclear translocation of YB-1. (A) Nuclear translocation of YB-1 with gemcitabine treatment was observed in 4 cancer cell lines. HeLa, MCF-7, 5637 and T24 cells were transfected with mCherry-YB-1. After $24 \mathrm{~h}$, all cell lines were treated with an anticancer drug. HeLa cells were treated with $13 \mu \mathrm{M}$ cisplatin (b) or $35 \mu \mathrm{M}$ gemcitabine (c). MCF-7, 5637 and T24 cells were treated with 5 and $10 \mu \mathrm{M}$ and $20 \mathrm{nM}$ gemcitabine (d, e and f), respecitively. (a) HeLa cells transfected with mCherry alone and treated with gemcitabine were used as control. Left images, before anticancer drug treatment (-). Right images, after anticancer drug treatment (+). Bars, $10 \mu \mathrm{m}$. (B) Nuclear translocation of endogenous YB-1 was increased relatively rapidly and was maintained for up to $4 \mathrm{~h}$ after gemcitabine treatment. The 5637 cells were treated for the indicated time with $10 \mu \mathrm{M}$ gemcitabine following which the cells were fixed and stained with anti-YB-1 antibody. The middle line of the box is the median. Brackets with asterisks indicate statistically significant differences between the data sets based on the Steel's test. ${ }^{*} \mathrm{P}<0.05,{ }^{* *} \mathrm{P}<0.01$.

translocates to the nucleus with expression of p53 in CIS and thereby increases p-glycoprotein expression.

Effect of p53 on YB-1 nuclear translocation. To investigate whether p53 is involved in the nuclear translocation of YB-1, we introduced exogenous P53 into a human cancer cell line and then observed the cellular localization of YB-1. First, we assessed the expression levels of p53 in the HeLa, T24 and 5637 cells by western blotting. It is known that the expression level of the endogenous p53 protein is suppressed to a low level in HeLa cells (Fig. 2A) because of its ubiquitination and degradation by the E6 protein expressed in human papilloma virus transformed HeLa cells (19). T24 cells contain a P53 mutant that has an in-frame deletion of tyrosine 126 and this mutant p53 protein is expressed at a low level compared to cancer cell lines with wild-type P53. The 5637 cells contain a $P 53$ point mutant $(\mathrm{R} 280 \mathrm{~T})$ and overexpression of the $\mathrm{p} 53$ protein may correlate with P53 mutation $(20,21)$. As expected, in western blotting the p53 protein was undetectable in HeLa and T24 cells, whereas high expression of p53 was seen in the 5637 cells (Fig. 2A). Next, we introduced P53 into HeLa cells. Thirty-six hours after transfection of pEGFP-wild-type p53, some of the YB-1 population had translocated to the nucleus (Fig. 2B) and the nuclear/cytoplasmic (N/C) ratio was increased compared to that of control cells (Fig. 2C). Moreover, introduction of a mutant p53 (R273H), which is known to lead to resistance to a variety of anticancer drugs, increased the $\mathrm{N} / \mathrm{C}$ ratio compared to that of control or cells in which wildtype p53 had been introduced (22). These data suggested that p53 coordinates the nuclear translocation of YB-1 and that a high amount of p53 protein is essential for this translocation.

Drug-induced YB-1 nuclear translocation and p-glycoprotein expression. Next, we examined whether gemcitabine induces the nuclear translocation of YB-1. First, HeLa cells were transfected with mCherry-fused YB-1 (mCherry-YB-1) and treated with gemcitabine. Treatment with gemcitabine $(35 \mu \mathrm{M})$ or with cisplatin $(13 \mu \mathrm{M})$ induced translocation of mCherryYB-1 to the nucleus (Fig. 3A) (23). Nuclear translocation of mCherry-YB-1 with gemcitabine treatment was also observed in the breast cancer MCF-7 cells and in the urinary bladder cancer 5637 and T24 cells, indicating that this phenomenon was not cell-specific. The nuclear translocation of YB-1 was maintained for up to $4 \mathrm{~h}$ after gemcitabine treatment. An immunohistochemical analysis in the 5637 cells showed that endogenous YB-1 also accumulated in the nucleus with 


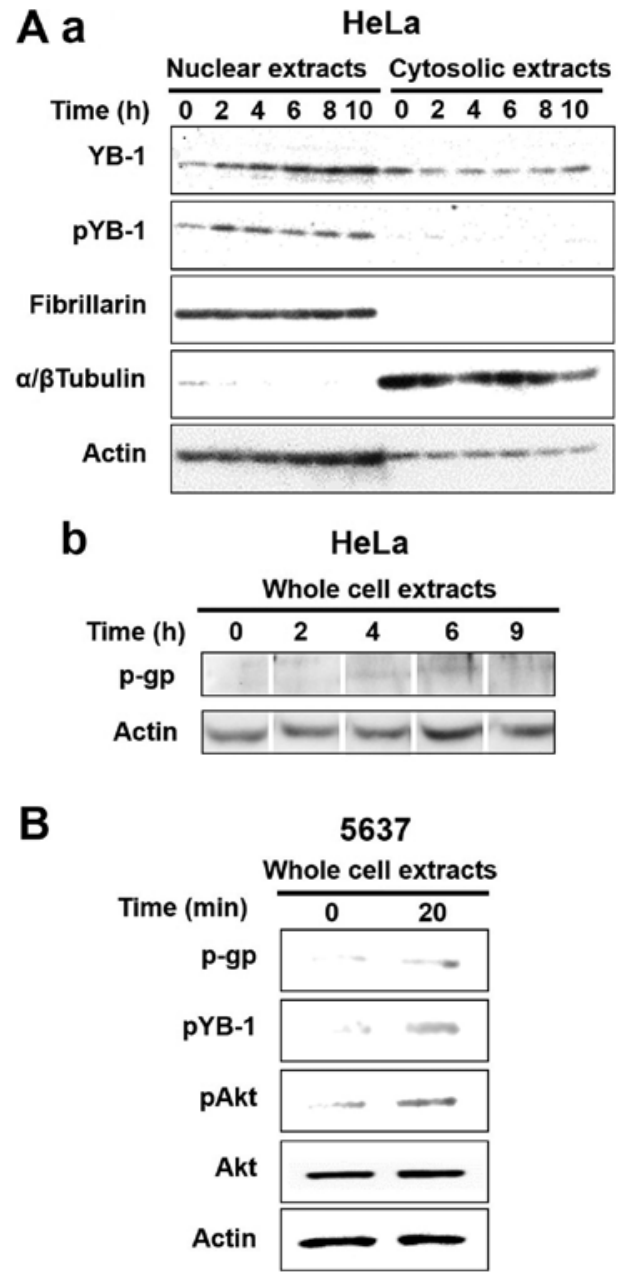

Figure 4. Both nuclear YB-1 and P-glycoprotein were increased after gemcitabine treatment. (A) Subcellular localization of endogenous YB-1 (a) and P-glycoprotein expression (b) were analyzed by western blotting at the indicated times after gemcitabine treatment. HeLa cells were treated with $35 \mu \mathrm{M}$ gemcitabine for the indicated times following which the cells were fractionated into nuclear and cytosolic components for analysis of YB-1 (a) or were extracted to whole cell lysate for analysis of P-glycoprotein (b). Nuclear extracts $(20 \mu \mathrm{g})$, cytoplasmic extracts $(40 \mu \mathrm{g})$ and whole cell extracts $(20 \mu \mathrm{g})$ were applied to each well for SDS-PAGE and electroblotted membranes were stained with anti-YB-1 (a, top), anti-pYB-1 (a, 2nd from the top), anti-Fibrillarin (a, 3rd from the top; control for nuclear extracts), anti- $\alpha / \beta$ tubulin ( $a, 4$ th from the top; control for cytosolic extracts), anti-actin (a and b, bottom; loading control) and anti-P-glycoprotein (b, top) antibodies. (B) P-glycoprotein protein expression of the bladder cancer cell line 5637 was analyzed by western blotting at the indicated times after gemcitabine treatment. The 5637 cells were treated with $10 \mu \mathrm{M}$ gemcitabine for the indicated times following which cell extracts were made and $20 \mu \mathrm{g}$ of the total extracted proteins were applied to each well of a gel for SDS-PAGE. Electroblotted membranes were stained with anti-P-glycoprotein (p-gp), anti-pYB-1, anti-pAkt, anti-Akt and anti-actin (loading control) antibodies.

gemcitabine treatment (Fig. 3B), while YB-1 was not accumulated in T24 cells (data not shown). Since Akt-mediated phosphorylation of YB-1 at Ser102 is required for nuclear translocation of YB-1 (24), we checked the phosphorylation status of YB-1 in the nuclear fraction of gemcitabine-treated cells. The phosphorylation of nuclear YB-1 was increased $2 \mathrm{~h}$ after gemcitabine treatment (Fig. 4A-a). Furthermore, the increase of the phosphorylated YB-1 corresponded to an increase in P-glycoprotein expression (Fig. 4A-b). In addition, Akt-induced YB-1 mediated P-glycoprotein expression
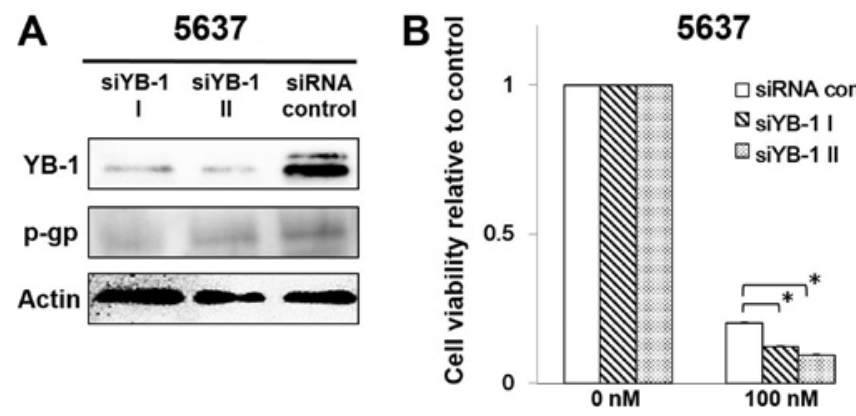

Figure 5. YB-1 knockdown decreased P-glycoprotein expression and sensitized the 5637 cells to gemcitabine. (A) YB-1 knockdown decreased P-glycoprotein expression. At 3 days after siRNA transfection of 5637 cells, total proteins were extracted from the cells and used for analysis of YB-1 and p-glycoprotein expression by western blotting. Total extracted proteins $(40 \mu \mathrm{g})$ were applied to each well of a gel for SDS-PAGE and electroblotted membranes were stained with anti-P-glycoprotein (p-gp), anti-YB-1 and anti-actin (loading control) antibodies. (B) The 5637 cells transfected with YB-1 siRNA showed decreased resistance to gemcitabine compared with control. At 2 days after siRNA transfection of 5637 cells, they were seeded into 96 -well plates. The following day, gemcitabine $(100 \mathrm{nM})$ were applied. After $72 \mathrm{~h}$, the cell survival rates were analyzed by cytotoxicity analysis. Brackets with asterisks indicate statistically significant differences between the data sets based on a Wilcoxon signed-rank test. ${ }^{*} \mathrm{P}<0.05$.

was more increased in the gemcitabine treated 5637 cells vs. control cells (Fig. 4B).

YB-1 knockdown decreased P-glycoprotein expression and sensitized 5637 cells to gemcitabine. To examine the effect of YB-1 knockdown on P-glycoprotein expression, the 5637 cells were transfected with siRNA YB-1 I, siRNA YB-1 II or siRNA control. Western blotting indicated that $\mathrm{P}$-glycoprotein expression was significantly decreased in YB-1 silenced cells compared with the siRNA transfected control cells (at 3 days after siRNA transfection) (Fig. 5A). Moreover, the 5637 cells transfected with YB-1 siRNA showed decreased resistance to gemcitabine compared with the control (Fig. 5B).

\section{Discussion}

We present data indicating that YB-1 translocation to the nucleus correlates significantly with p53 expression in CIS. Nuclear translocation of YB-1 is thought to be involved in drug resistance by increasing MDR-1 transcription and its product, P-glycoprotein expression (9). The present study showed that YB-1 siRNA transfected into a bladder cancer cell line decreased P-glycoprotein expression is consistent with the involvement of YB-1 in P-glycoprotein expression. Indeed, our data of clinical samples showed that nuclear expression of YB-1 correlated with the expression of P-glycoprotein. Moreover, YB-1 translocation to the nucleus correlates significantly with p53 expression in CIS. Nuclear expression of YB-1 was observed more often in p53-positive cases than in p53 negative cases (41 and 5\%, respectively). These data indicated that p53 may regulate the nuclear transport of YB-1 in bladder CIS.

Further we showed that the $\mathrm{R} 273 \mathrm{H}$ mutation of $\mathrm{p} 53$ induced more YB-1 nuclear translocation compared to wild-type $\mathrm{p} 53$ in vitro. The $\mathrm{R} 273 \mathrm{H}$ mutation of $\mathrm{p} 53$ is thought to be a 'gain-of-function' mutation that drives oncogenesis (25). 
Zhang et al (15) reported that some tumor-derived mutants of p53 including $\mathrm{R} 273 \mathrm{H}$ also induce nuclear translocation of YB-1 and that nuclear expression of YB-1 is not seen in p53 null cell lines. These data are consistent with recent reports that p53 upregulates YB-1 nuclear import in different cell types $(11,15-17)$. Nuclear accumulation of p53 in bladder cancer is thought to be a result of mutation of p53, including the $\mathrm{R} 273 \mathrm{H}$ mutation $(26,27)$. Combined with these previous reports, our findings indicate that urinary CIS may acquire the property of drug resistance during its oncogenesis through overexpression of p53 or mutation of p53.

Third, we showed that gemcitabine induced both nuclear translocation of YB-1 and increased the expression of P-glycoprotein and, in addition, YB-1 knockdown increased the effect of gemcitabine in bladder cancer. To the best of our knowledge, this is the first report that YB-1 is involved in gemcitabine chemoresistance in bladder cancer. We showed that gemcitabine treatment increased the nuclear translocation of YB-1 in 5637 cells that have a high expression level of p53, but not in T24 cells that have a low expression level of p53. These results support the notion that a high expression level of mutant p53 protein is necessary for nuclear translocation of YB-1 in bladder cancer. Nowadays, GC therapy is a standard therapy for aggressive bladder cancer. However, gemcitabine-induced drug resistance in bladder cancer compromises the therapeutic efficacy. Although it has been proposed that genes required for gemcitabine transport and metabolism such as human equilibrative nucleoside transporter-1 and deoxycytidine kinase are involved in the mechanism of cellular resistance to gemcitabine, the mechanisms by which gemcitabine resistance occurs are not fully understood $(28,29)$.

Finally, gemcitabine has recently been used for intravesical chemotherapy and it is expected that gemcitabine will be more frequently used for bladder cancer treatment in the future. Our in vitro findings indicate that further investigation of the association of p53 expression and P53 gene status and YB-1 expression in bladder cancer with drug-resistance, including gemcitabine resistance, is warranted.

\section{Acknowledgements}

The present study was supported by a Grant-in-Aid for Young doctor of SMC (grant no. 26-F-1-05). We thank Kazuko Matsuno, Yuko Ohno, Kumiko Ohsawa and Tomoaki Aoki for their technical assistance, and Akiko Murata of Leica Microsystems for technical advice regarding immunohistochemistry.

\section{References}

1. Zieger K, Marcussen N, Borre M, Orntoft TF and Dyrskjot L: Consistent genomic alterations in carcinoma in situ of the urinary bladder confirm the presence of two major pathways in bladder cancer development. Int J Cancer 125: 2095-2103, 2009.

2. Knowles MA and Hurst CD: Molecular biology of bladder cancer: New insights into pathogenesis and clinical diversity. Nat Rev Cancer 15: 25-41, 2015.

3. Lamm D, Herr H, Jakse G, Kuroda M, Mostofi FK, Okajima E, Sakamoto A, Sesterhenn I and da Silva FC: Updated concepts and treatment of carcinoma in situ. Urol Oncol 4: 130-138, 1998.
4. Parker WP, Ho PL, Melquist JJ, Scott K, Holzbeierlein JM, LopezCorona E, Kamat AM and Lee EK: The effect of concomitant carcinoma in situ on neoadjuvant chemotherapy for urothelial cell carcinoma of the bladder: Inferior pathological outcomes but no effect on survival. J Urol 193: 1494-1499, 2015.

5. von der Maase H, Hansen SW, Roberts JT, Dogliotti L, Oliver T, Moore MJ, Bodrogi I, Albers P, Knuth A, Lippert CM, et al: Gemcitabine and cisplatin versus methotrexate, vinblastine, doxorubicin, and cisplatin in advanced or metastatic bladder cancer: Results of a large, randomized, multinational, multicenter, phase III study. J Clin Oncol 18: 3068-3077, 2000.

6. Shelley MD, Jones G, Cleves A, Wilt TJ, Mason MD and Kynaston HG: Intravesical gemcitabine therapy for non-muscle invasive bladder cancer (NMIBC): A systematic review. BJU Int 109: 496-505, 2012.

7. Uchiumi T, Kohno K, Tanimura H, Hidaka K, Asakuno K, Abe $\mathrm{H}$, Uchida $\mathrm{Y}$ and Kuwano $\mathrm{M}$ : Involvement of protein kinase in environmental stress-induced activation of human multidrug resistance 1 (MDR1) gene promoter. FEBS Lett 326: 11-16, 1993.

8. Asakuno K, Kohno K, Uchiumi T, Kubo T, Sato S, Isono M and Kuwano M: Involvement of a DNA binding protein, MDR-NF1/ YB-1, in human MDR1 gene expression by actinomycin D. Biochem Biophys Res Commun 199: 1428-1435, 1994.

9. Ohga T, Uchiumi T, Makino Y, Koike K, Wada M, Kuwano M and Kohno K: Direct involvement of the Y-box binding protein YB-1 in genotoxic stress-induced activation of the human multidrug resistance 1 gene. J Biol Chem 273: 5997-6000, 1998.

10. Koike K, Uchiumi T, Ohga T, Toh S, Wada M, Kohno K and Kuwano M: Nuclear translocation of the Y-box binding protein by ultraviolet irradiation. FEBS Lett 417: 390-394, 1997.

11. Okamoto T, Izumi H, Imamura T, Takano H, Ise T, Uchiumi $\mathrm{T}$, Kuwano $\mathrm{M}$ and Kohno K: Direct interaction of p53 with the Y-box binding protein, YB-1: A mechanism for regulation of human gene expression. Oncogene 19: 6194-6202, 2000.

12. Stein U, Jürchott K, Walther W, Bergmann S, Schlag PM and Royer HD: Hyperthermia-induced nuclear translocation of transcription factor $\mathrm{YB}-1$ leads to enhanced expression of multidrug resistance-related ABC transporters. J Biol Chem 276: 28562-28569, 2001.

13. Holm PS, Bergmann S, Jurchott K, Lage H, Brand K, Ladhoff A, Mantwill K, Curiel DT, Dobbelstein M, Dietel M, et al: YB-1 relocates to the nucleus in adenovirus-infected cells and facilitates viral replication by inducing E2 gene expression through the E2 late promoter. J Biol Chem 277: 10427-10434, 2002.

14. Sutherland BW, Kucab J, Wu J, Lee C, Cheang MC, Yorida E, Turbin D, Dedhar S, Nelson C, Pollak M, et al: Akt phosphorylates the Y-box binding protein 1 at Ser102 located in the cold shock domain and affects the anchorage-independent growth of breast cancer cells. Oncogene 24: 4281-4292, 2005.

15. Zhang YF, Homer C, Edwards SJ, Hananeia L, Lasham A, Royds J, Sheard P and Braithwaite AW: Nuclear localization of Y-box factor YB1 requires wild-type p53. Oncogene 22: 2782-2794, 2003.

16. Guay D, Gaudreault I, Massip L and Lebel M: Formation of a nuclear complex containing the p53 tumor suppressor, YB-1, and the Werner syndrome gene product in cells treated with UV light. Int J Biochem Cell Biol 38: 1300-1313, 2006.

17. Homer C, Knight DA, Hananeia L, Sheard P, Risk J, Lasham A, Royds JA and Braithwaite AW: Y-box factor YB1 controls p53 apoptotic function. Oncogene 24: 8314-8325, 2005.

18. Birbach A, Gold P, Binder BR, Hofer E, de Martin R and Schmid JA: Signaling molecules of the NF-kappa B pathway shuttle constitutively between cytoplasm and nucleus. J Biol Chem 277: 10842-10851, 2002.

19. Thomas M, Pim D and Banks L: The role of the E6-p53 interaction in the molecular pathogenesis of HPV. Oncogene 18: 7690-7700, 1999.

20. Cooper MJ, Haluschak JJ, Johnson D, Schwartz S, Morrison LJ, Lippa M, Hatzivassiliou G and Tan J: p53 mutations in bladder carcinoma cell lines. Oncol Res 6: 569-579, 1994.

21. Hinata N, Shirakawa T, Zhang Z, Matsumoto A, Fujisawa M, Okada H, Kamidono $\mathrm{S}$ and Gotoh A: Radiation induces p53-dependent cell apoptosis in bladder cancer cells with wildtype-p53 but not in p53-mutated bladder cancer cells. Urol Res 31: 387-396, 2003. 
22. Wong RP, Tsang WP, Chau PY, Co NN, Tsang TY and Kwok TT: p53-R273H gains new function in induction of drug resistance through down-regulation of procaspase-3. Mol Cancer Ther 6: 1054-1061, 2007.

23. Ahmed M and Jamil J: Cytotoxicity of neoplastic drugs Gefitinib, Cisplatin, 5-FU, Gemcitabine, and Vinorelbine on human cervical cancer cells (HeLa). Biol Med 3: 60-71, 2012.

24. Evdokimova V, Ruzanov P, Anglesio MS, Sorokin AV, Ovchinnikov LP, Buckley J, Triche TJ, Sonenberg N and Sorensen PH: Akt-mediated YB-1 phosphorylation activates translation of silent mRNA species. Mol Cell Biol 26: 277-292, 2006.

25. Zhu J, Sammons MA, Donahue G, Dou Z, Vedadi M, Getlik M, Barsyte-Lovejoy D, Al-awar R, Katona BW, Shilatifard A, et al: Gain-of-function p53 mutants co-opt chromatin pathways to drive cancer growth. Nature 525: 206-211, 2015.

26. Esrig D, Spruck CH III, Nichols PW, Chaiwun B, Steven K Groshen S, Chen SC, Skinner DG, Jones PA and Cote RJ: p53 nuclear protein accumulation correlates with mutations in the p53 gene, tumor grade, and stage in bladder cancer. Am J Pathol 143: 1389-1397, 1993.
27. Grimm MO, Jürgens B, Schulz WA, Decken K, Makri D and Schmitz-Dräger BJ: Inactivation of tumor suppressor genes and deregulation of the c-myc gene in urothelial cancer cell lines. Urol Res 23: 293-300, 1995

28. Matsumura N, Nakamura Y, Kohjimoto Y, Inagaki T, Nanpo Y, Yasuoka H, Ohashi Y and Hara I: The prognostic significance of human equilibrative nucleoside transporter 1 expression in patients with metastatic bladder cancer treated with gemcitabinecisplatin-based combination chemotherapy. BJU Int 108: E110-E116, 2011.

29. Nakano Y, Tanno S, Koizumi K, Nishikawa T, Nakamura K, Minoguchi M, Izawa T, Mizukami Y, Okumura T and Kohgo Y: Gemcitabine chemoresistance and molecular markers associated with gemcitabine transport and metabolism in human pancreatic cancer cells. Br J Cancer 96: 457-463, 2007. 